Revista Interdisciplinaria de Humanidades, Educación, Ciencia y Tecnología

Año VI. Vol. VI. N¹0. Enero - Julio 2020

Hecho el depósito de ley: pp201602FA4721

ISSN-L: 2542-3029; ISSN: 2610-802X

Universidad Nacional Experimental Francisco de Miranda (UNEFM). Santa Ana de Coro. Venezuela

Pedro Cruz

DOI 10.35381/cm.v6i10.244

\title{
Gestión del conocimiento para el desarrollo de la capacidad del estudiante de educación primaria de Colombia
}

\section{Knowledge Management in the capacity development of the Colombian primary education student}

\author{
Pedro Cruz \\ dropecruz@hotmail.com \\ Institución Educativa San Alberto Magno \\ Colombia \\ https://orcid.org/0000-0002-3052-4660
}

Recibido: 1 de junio de 2019

Aprobado: 1 de julio de 2019

\begin{abstract}
RESUMEN
El objeto de esta investigación fue estudiar la Gestión del Conocimiento como alternativa dentro del desarrollo organizacional para el Desarrollo de la capacidad del estudiante de Educación Primaria de Colombia. Respaldada en los postulados teóricos de Pavez (2009), Finol y Camacho (2008), entre otros, enmarcada dentro del paradigma de una investigación cualitativa, con información recolectada por medio de entrevistas semiestructuradas, empleando como técnica la observación directa. Como muestra se tomarán (5) actores sociales, conformados por estudiantes y docentes de de las instituciones INESAM-Cesar Colombia. Obteniendo como resultado principal que la gestión del conocimiento constituye un recurso valioso dentro de las instituciones educativas, por cuanto permite el desarrollo del aprendizaje organizacional de los miembros que lo conforman, permitiendo valorar los activos tangibles, por medio de procesos dinámicos de creación, asimilación, difusión y utilización del conocimiento que van a representar la competitividad dentro de la misma.
\end{abstract}

Descriptores: Gestión; Conocimiento; Desarrollo; Capacidad; Educación Primaria.

\section{ABSTRACT}

The purpose of this research was to analyze the management of knowledge in the development of the capacity of the primary education student in Colombia. Backed in the theoretical postulates of Pavez (2009), Finol and Camacho (2008), among others; It is framed as a descriptive, field, transectional and non-experimental research. The 


\section{CIENCIAMATRIA}

Revista Interdisciplinaria de Humanidades, Educación, Ciencia y Tecnología

Año VI. Vol. VI. N¹0. Enero - Julio 2020

Hecho el depósito de ley: pp201602FA4721

ISSN-L: 2542-3029; ISSN: 2610-802X

Universidad Nacional Experimental Francisco de Miranda (UNEFM). Santa Ana de Coro. Venezuela

\section{Pedro Cruz}

population consisted of (55) subjects of the La Curva - Cesar and INESAM - Cesar Colombia institutions. For the collection of the information, the observation technique was used by means of a survey, a questionnaire-type instrument made up of (48) items, validated through the expert judgment, a pilot test was carried out on 10 subjects, the Cronbach Alpha coefficient was applied with a reliability index of 0.90 . For the analysis of the data, descriptive statistics were used, indicating the frequency in the responses and the arithmetic mean by dimension and indicators. It was concluded that in these educational institutions, knowledge management in their teachers is of medium presence.

Descriptors: Management; Knowledge; Developing; Capacity; Primary education.

\section{INTRODUCCIÓN}

Los estudios sobre el conocimiento datan desde los inicios de la humanidad, afianzándose en los filósofos estudiosos del pensamiento humano entre los cuales se distingue Platón con su teoría de las ideas, quien concibe el pensamiento como el punto culminante de un proceso ascendente, que con el apoyo de la ciencia y el razonamiento accede al mundo de las ideas y es transferible a la auténtica realidad.

Siguiendo esta línea de pensamiento, la gestión del conocimiento visto como un paradigma dentro de las organizaciones públicas y privadas, ha venido en una evolución constante infiltrándose a través de la red de formación a nivel mundial, donde su implementación ha sido de alto beneficio dentro de las organizaciones, en América latina el despliegue de la gestión del conocimiento ha sido un tanto moderado en su aplicación dentro de las organizaciones, dirigido más a investigaciones, análisis y disertaciones sobre la materia.

Por ello, es necesaria la formación del talento humano para buscar sus respuestas y plantear sus propias interrogantes con la finalidad de diseñar sus propios experimentos y así generar conocimientos. Aquí adquiere mayor importancia el conocimiento constituido por los actores en el proceso de formación. En ese sentido, el aprendizaje adquiere mayor relevancia. Por tanto, sin producción o generación de conocimiento no puede existir un aprendizaje consono con las exigencias del mundo cambiante, es decir, se hace necesaria polivalencia, flexibilidad y capacidad para aprender permanentemente. Es así como, para Mayo y Lank (2010) son muy pocas 


\section{CIENCIAMATRIA}

Revista Interdisciplinaria de Humanidades, Educación, Ciencia y Tecnología

Año VI. Vol. VI. N¹0. Enero - Julio 2020

Hecho el depósito de ley: pp201602FA4721

ISSN-L: 2542-3029; ISSN: 2610-802X

Universidad Nacional Experimental Francisco de Miranda (UNEFM). Santa Ana de Coro. Venezuela

\section{Pedro Cruz}

las organizaciones educativas o empresariales que pueden afirmar que cuentan con una capacidad adecuada para gestionar la información y el conocimiento que pretenden llevar a las personas con las que tratan, siendo necesario contar con instituciones que gestionen un conocimiento en pertinencia a los retos globales, con la finalidad de generar una praxis que permita actualizarse en función de los principios de una organziació compleja que aprende haciendo, lanni Gómez (2017). En ese sentido entonces las instituciones se han dado a la tarea de acoplar sus redes internas de trabajo a lo que el manejo de la información y transferencia de conocimiento se refiere, ya que al hacer grandes inversiones en tecnología han tenido que modificar estrategias para que el trabajo de verdad resulte productivo. Así, como lo expone Nordan (2009), cuando indica que la generación de conocimiento parte primeramente de entender el proceso de creación del conocimiento, además de ver en qué áreas se debe ayudar. Luego es necesario mantener las cosas simples, no ser tan ambicioso como para proponer un gran cambio en las instituciones educativas, porque los proyectos que llevan varios años casi nunca son completados.

El proyecto que solucione un problema importante en las instituciones, es de gran ayuda para ganar seguidores que se comprometan con la creación de conocimiento. Por ende, se hace necesario que las personas tengan las suficientes competencias para poder asumir los proyectos ya que, si se percibe alguna falla en cualquier acción a tomar, entonces se pierde la credibilidad sobre todo en el conocimiento que se está generando a las personas que están sirviendo en este caso los usuarios.

\section{Desarrollo Teórico}

\section{Gestión del conocimiento}

La variable dispuesta para el desarrollo de esta investigación es la gestión del conocimiento en el desarrollo de la capacidad del estudiante que para el caso de las instituciones de educación primaria de Colombia, será estudiado en dirección a las posibilidades que brinda el actual esquema del capital intelectual de estas entidades. Sobre la gestión del conocimiento, diversas son las metodologías que se pueden plantear, a causa de los diversos enfoques y escuelas que existen en la actualidad. 


\section{CIENCIAMATRIA}

Revista Interdisciplinaria de Humanidades, Educación, Ciencia y Tecnología

Año VI. Vol. VI. N¹0. Enero - Julio 2020

Hecho el depósito de ley: pp201602FA4721

ISSN-L: 2542-3029; ISSN: 2610-802X

Universidad Nacional Experimental Francisco de Miranda (UNEFM). Santa Ana de Coro. Venezuela

\section{Pedro Cruz}

Para Nonaka y Takeuchi, (2005), la gestión del conocimiento (GC) es definida como la capacidad de la empresa para crear conocimiento nuevo, diseminarlo en la organización e incorporarlo en productos, servicios y sistemas. Esta definición implica que la gestión del conocimiento (GC) integra un complejo rango de actividades que abarca, desde la creación o captación, estructuración, transformación y transferencia de conocimiento, hasta su almacenamiento e incorporación a todos los procesos.

Así también, a juicio de Pavez (2009), se trata igualmente del proceso mediante el cual se obtiene, despliega o utiliza una variedad de recursos básicos para apoyar los objetivos de la organización. Desde este punto de vista la gestión del conocimiento debe cumplir con el concepto anteriormente expresado, entendiendo como recursos al conocimiento. Por ello, la gestión del conocimiento permite que el logro del saber se disponga para el crecimiento organizacional.

Por otra parte, Brooking (2009), lo concibe como "la actividad que se preocupa de la estrategia y la táctica para gestionar activos centrados en las personas", mientras que Bueno (2010 p. 76), la ve como "la función que planifica, coordina y controla los flujos de conocimiento que se producen en la empresa en relación con sus actividades y con su entorno a fin de crear unas competencias básicas esenciales".

Del mismo modo, Andreu y Sieber (2009), también definen la gestión del conocimiento como el proceso que continuamente asegura el desarrollo y aplicación de todo tipo de conocimientos pertinentes en una empresa, con objeto de mejorar su capacidad de resolución de problemas y así contribuir a la sostenibilidad de sus ventajas competitivas.

En este sentido, es importante gestionar el conocimiento que surge dentro de las organizaciones (también utilizando el conocimiento que viene desde el exterior de ésta), sumado a que los cambios constantes, vertiginosos y profundos que suceden en la sociedad hacen también que ciertos conocimientos se vuelvan pronto obsoletos, con lo cual aprender y desaprender se torna una actividad urgente. De ahí que en el caso de las instituciones de educación primaria de Colombia la gestión del conocimiento debe ser impulsada hacia la intención de cambio y superación. 


\section{CIENCIAMATRIA}

Revista Interdisciplinaria de Humanidades, Educación, Ciencia y Tecnología

Año VI. Vol. VI. N¹0. Enero - Julio 2020

Hecho el depósito de ley: pp201602FA4721

ISSN-L: 2542-3029; ISSN: 2610-802X

Universidad Nacional Experimental Francisco de Miranda (UNEFM). Santa Ana de Coro. Venezuela

Pedro Cruz

\section{Componentes de gestión del conocimiento}

Los modelos o componentes básicos se construyen con base a conceptos $\mathrm{u} / \mathrm{o}$ relaciones y deben ser formales, para ello, se requiere de modelos operativos que establezcan planteamientos comprobados y estructurados para formar un sistema que dé como resultado una teoría, siendo esta la estructura abstracta que representa la realidad. De alguna manera, el modelo o componente se da en un marco teórico general es decir hace referencia a ese campo general.

De acuerdo con Yurén $(2009$, p. 323), "el modelo relaciona lo abstracto con lo concreto". Es decir, que forzosamente el modelo o componente deba ser algo material para ser manejado con las manos. Al especificar la teoría se pasa de lo abstracto a lo concreto los aspectos fundamentales proporcionados por la teoría. Sin embargo, en el modelo las contradicciones han sido superadas por los conceptos ya son transferibles mediante lógica consistente y sistemática.

Al revisar lo planteado por, Klaus y Rivas (2008, p. 94), al referirse a los modelos o componentes, plantean "que un modelo es más inmediatamente perceptible en una metáfora o una analogía". Puede decirse en referencia a lo expuesto, un modelo es una construcción teórico hipotética que puede hacerse praxis al representar a un sector de la realidad, susceptible de estudio y verificación lo que implica que además de representar la teoría debe evidenciar las condiciones en las que se produce un fenómeno al establecer la conexión de lo abstracto con lo concreto.

En consecuencia, se puede señalar que la calidad es la correspondencia entre el ser y el quehacer de la institución educativa con su deber ser. Así mismo, los modelos contenidos en una teoría forman un conjunto de relaciones y conceptos que son tan bien modelos formales, que pueden ser verbales, gráficos, matemáticos y materiales.

De la misma manera, Valhondo (2009, p. 67), acota que "el concepto académico tradicional de la calidad está supeditado al rendimiento de logros intelectuales sobresalientes en el avance del conocimiento", también la conciben como el cumplimiento de una misión eficaz y eficiente en la operatividad de los objetivos que 


\section{CIENCIAMATRIA}

Revista Interdisciplinaria de Humanidades, Educación, Ciencia y Tecnología

Año VI. Vol. VI. N¹0. Enero - Julio 2020

Hecho el depósito de ley: pp201602FA4721

ISSN-L: 2542-3029; ISSN: 2610-802X

Universidad Nacional Experimental Francisco de Miranda (UNEFM). Santa Ana de Coro. Venezuela

\section{Pedro Cruz}

den satisfacción al cliente, premiando así el valor de la inversión.

En referencia a lo anterior, puede decirse que el concepto académico tradicional de la calidad está supeditado al rendimiento de logros intelectuales sobresalientes en el avance del conocimiento es decir que se hace necesario poner en práctica todas las cualidades intelectuales del individuo para lograr un buen desempeño.

\section{Proceso de desarrollo del conocimiento organizacional}

Las organizaciones se desarrollan actualmente en un entorno que se expande a un nivel cada vez más acelerado, interdependiente, impredecible, dinámico y complejo; donde el estado de cambio es permanente. Por tal razón, las organizaciones deben ser ágiles, capaces y orientarse al desarrollo de capacidades de aprendizaje y de conocimiento organizacional.

El proceso de desarrollo del conocimiento en la organización educativa debe dirigirse a la creación de conocimientos esenciales. Esto se debe a que no todos los conocimientos que poseen los recursos humanos y la organización son conocimientos esenciales, así como tampoco lo son todos lo que se encuentran en el entorno.

La organización educativa para su desempeño exitoso debe aprender, y aprender exige una correcta transmisión del conocimiento esencial en su interior, tanto el que se desarrolla por medio de la interacción continua entre los miembros de la organización, como el que se desarrolla entre éstos y el ambiente. Un aspecto clave para la creación de conocimientos esenciales es el proceso de conversión del conocimiento; de tácito a tácito, de tácito a explícito, de explícito a tácito, de explícito a explícito. Este proceso permite igualmente el flujo de conocimiento, y proporciona además transparencia y dinamismo a los activos esenciales.

En ese sentido, para Prieto (2013), la dinámica del conocimiento es el resultado de un proceso de intercambio entre los estímulos del entorno, los conocimientos que existen en el sistema interno de la organización educativa y las acciones de sus integrantes; donde esos conocimientos y acciones son la entrada y salida del flujo de conversión de conocimiento. 


\section{CIENCIAMATRIA}

Revista Interdisciplinaria de Humanidades, Educación, Ciencia y Tecnología

Año VI. Vol. VI. N¹0. Enero - Julio 2020

Hecho el depósito de ley: pp201602FA4721

ISSN-L: 2542-3029; ISSN: 2610-802X

Universidad Nacional Experimental Francisco de Miranda (UNEFM). Santa Ana de Coro. Venezuela

\section{Pedro Cruz}

La relevancia de la dinámica del conocimiento radica en las habilidades que debe poseer la organización para desarrollar, transformar y aprovechar este activo para crear un estado de equilibrio permanente con el entorno. Estas habilidades se adquieren por medio del aprendizaje, que cada organización es capaz de desarrollar; el aprendizaje es una capacidad organizacional que puede desarrollarse y es de gran utilidad para mantener de forma estable los retos que propician las exigencias del entorno y los activos del conocimiento.

Por su parte, Probst, Raub y Romhardt (2011) consideran que el aprendizaje organizacional se soporta en los cambios que se realizan en sus bases del conocimiento, la creación de marcos de referencia colectivos y el desarrollo de las aptitudes para actuar y solucionar problemas. Seguidamente, conceptualizan la base del conocimiento de una organización como los activos intelectuales, individuales y colectivos, que ella puede utilizar para realizar sus actividades.

El aprendizaje organizacional entonces viene a constituir un proceso sistemático e indispensable para su funcionamiento, el aprendizaje puede ser una cualidad intrínseca de cualquier sistema organizacional y potencia las capacidades que condicionan la efectividad de la organización.

La esencia de dicho aprendizaje radica en que la organización debe ser vista por sus miembros como un sistema abierto; cada uno de ellos debe ser capaz de integrar las funciones de hacer y pensar a todos los niveles organizacionales. También posibilita que sean capaces de percibir e interpretar los acontecimientos que puedan surgir en el entorno, así como actuar en beneficio mutuo.

El aprendizaje organizacional posibilita perfeccionar de forma efectiva, el conocimiento esencial que fluye dentro y fuera de los límites de la organización; además posibilita mejorar las capacidades organizacionales. Estas capacidades se logran por medio de la creación de procesos que contribuyen al flujo de conocimiento entre los miembros de la organización, y de éstos con el entorno.

La organización alcanza por medio del aprendizaje un nivel de equilibrio con las oportunidades y amenazas que presenta el medio, el aprendizaje es, a su vez, un proceso de interacción con el medio, tanto desde el punto de vista de adaptación 


\section{CIENCIAMATRIA}

Revista Interdisciplinaria de Humanidades, Educación, Ciencia y Tecnología

Año VI. Vol. VI. N¹0. Enero - Julio 2020

Hecho el depósito de ley: pp201602FA4721

ISSN-L: 2542-3029; ISSN: 2610-802X

Universidad Nacional Experimental Francisco de Miranda (UNEFM). Santa Ana de Coro. Venezuela

\section{Pedro Cruz}

como de operación. El proceso de aprendizaje en las organizaciones ocurre en la confrontación entre la organización y el medio y se sustenta en la dinámica de desarrollo de capacidades de aprendizaje que esta sea capaz de sostener.

La capacidad de aprendizaje de cada organización depende de la dinámica interna de conocimiento y de su funcionamiento, además del nivel de exigencia interna para adaptarse a las transformaciones que presenta el entorno.

La capacidad de aprendizaje es entendida por Prieto (2013) como el potencial dinámico de creación, asimilación, difusión y utilización del conocimiento por medio de numerosos flujos de conocimientos que hacen posible la capacitación y evolución de los stocks - el conjunto de todos los conocimientos esenciales- de conocimientos que capacitan a las organizaciones y a sus agentes de conocimientos para actuar intencionadamente en entornos cambiantes. Con el desarrollo de capacidades de aprendizaje de la organización, viene aparejado la capacidad que ella posea para absorber conocimiento. Esta habilidad le permite asimilar, valorar e integrar los conocimientos a sus necesidades de funcionamiento, esto implica además contar con una gerencia transformacional que motive a la generación de conocimientos como proceso dinamizador de la capacidad de la organización en promover la investigación como fuente generadora del conocimiento, Morgado, Aldana Zavala \& Isea Argüelles (2019).

\section{La Sociedad del Conocimiento y la Gestión del Conocimiento}

Desde que Bell (2001) definiera la sociedad del conocimiento han ido surgiendo y cobrando gran predicamento un conjunto de ideas, conceptos y expresiones que modelan su contenido verdadero y su alcance real en la comunidad científica. Es el caso, entre otras expresiones, de las siguientes: capital humano, capital intelectual, activos intangibles, proceso de aprendizaje, organización inteligente, aprendizaje organizativo, conocimiento explícito, conocimiento tácito, economía del conocimiento y gestión del conocimiento.

Los conjuntos de conceptos citados ponen el acento en la suma de saberes, de conocimientos $\mathrm{o}$ en el capital intangible que posee y desarrolla la sociedad, las 


\section{CIENCIAMATRIA}

Revista Interdisciplinaria de Humanidades, Educación, Ciencia y Tecnología

Año VI. Vol. VI. N¹0. Enero - Julio 2020

Hecho el depósito de ley: pp201602FA4721

ISSN-L: 2542-3029; ISSN: 2610-802X

Universidad Nacional Experimental Francisco de Miranda (UNEFM). Santa Ana de Coro. Venezuela

\section{Pedro Cruz}

organizaciones o las empresas. Dicho capital intangible (más conocido como capital intelectual) representa, en este final de siglo, el valor más importante para hacer frente al reto competitivo de nuestro tiempo y, en consecuencia, poder abordar con cierto éxito el desafío de entrar en el nuevo milenio.

La sociedad del conocimiento se caracteriza por la aparición continua de saberes nuevos, por el desarrollo permanente de las facultades intelectuales, todo ello concretado en una aceleración inusitada de la caducidad de los paradigmas dominantes en los años precedentes, de la obsolescencia de los métodos de análisis y de las técnicas empleadas para la observación de la realidad por investigadores, analistas, expertos y profesionales.

Estamos viviendo en una sociedad en la que están adquiriendo primacía los conocimientos teóricos y los conocimientos tácitos sobre cualquier otra clase de conocimiento; es decir son relevantes aquéllos que requieren de un determinado modelo mental y de unos procesos basados en la creación, en las ideas, en la abstracción y en la innovación, tal y como indican Chan Kim y Mauborgne (2007), el caso de los conocimientos teóricos, son importantes aquellos que son de difícil transmisión y comunicación, dado que se basan en la experiencia y en el talento idiosincrásico, en el arte, en el "saber hacer", y en el caso de los conocimientos tácitos y el explícito, donde:

- Conocimiento Tácito: Este es un conocimiento muy personal y difícil de plantear a través del lenguaje formal y, por lo tanto, difícil de transmitir y compartir con otros. Tiene sus raíces en lo más profundo de la experiencia individual, así como en los ideales, valores y emociones de cada persona.

- Conocimiento Explícito: Es aquel que puede expresarse a través del lenguaje formal; es decir, con palabras y números, y puede transmitirse y compartirse fácilmente, en forma de datos, fórmulas científicas, procedimientos codificados o principios universales. Se expresa normalmente en algún soporte físico (libros, CD ROMS, imágenes). 


\section{CIENCIAMATRIA}

Revista Interdisciplinaria de Humanidades, Educación, Ciencia y Tecnología

Año VI. Vol. VI. N¹0. Enero - Julio 2020

Hecho el depósito de ley: pp201602FA4721

ISSN-L: 2542-3029; ISSN: 2610-802X

Universidad Nacional Experimental Francisco de Miranda (UNEFM). Santa Ana de Coro. Venezuela

Pedro Cruz

\section{METODOLOGÍA}

La presente investigación se enmarca dentro del paradigma o tradición cualitativa, el cual es un movimiento que localiza al observador en el mundo, consiste en un conjunto de prácticas interpretativas que hacen al mundo visible. Estas prácticas transforman el mundo, lo convierten en una serie de representaciones, que incluyen las notas de campo, las entrevistas, conversaciones, fotografías, registros y memorias.

Es por ello que según Denzin y Lincoln (2005), la investigación cualitativa implica una aproximación interpretativa y naturalista del mundo. Esto significa que los investigadores cualitativos estudian las cosas en su contexto natural, intentando dar sentido o interpretar los fenómenos en función de los significados que las personas le dan. Asimismo, señalan que el enfoque cualitativo, "utiliza la recolección de datos sin medición numérica para descubrir o afirmar preguntas de investigación en el proceso de interpretación"

De la misma manera, Bisquerra (2009) explica que la metodología implementada es la cualitativa, propia del paradigma postpositivista, a través de ésta se describe e interpreta la realidad percibida desde el accionar del hombre inmerso en un determinado contexto social, con el interés científico de comprender el significado, así como transformarla, ello requiere del contacto directo del investigador con el objeto de estudio. Por su parte, teóricos como Strauss y Corbin (2002, p.13) indican que dentro de la misma, se explican "detalles complejos de algunos fenómenos, tales como sentimientos, procesos de pensamiento y emociones, difíciles de extraer o de aprehender por métodos de investigación más convencionales", es decir, por procedimientos no estadísticos.

En palabras de Tójar (2006), la metodología cualitativa supera la simple contraposición a los principios positivistas, para concretarse en una postura intencionada, en método para la construcción de conocimiento, el cual va mucho más allá del análisis de datos, pues este se crea en cada una de las fases del proceso de investigación. Por ello, ésta es conceptualizada con varias acepciones 


\section{CIENCIAMATRIA}

Revista Interdisciplinaria de Humanidades, Educación, Ciencia y Tecnología

Año VI. Vol. VI. N¹0. Enero - Julio 2020

Hecho el depósito de ley: pp201602FA4721

ISSN-L: 2542-3029; ISSN: 2610-802X

Universidad Nacional Experimental Francisco de Miranda (UNEFM). Santa Ana de Coro. Venezuela

\section{Pedro Cruz}

como: transdisciplinaria, interdisciplinaridad, humanista, creatividad, ética, holística, multimetódica, entre otras; características éstas que van definiendo el perfil y roles del investigador.

En razón de lo antes expuesto, la recolección de los datos se hizo por medio de un instrumento denominado entrevistas cualitativas, que según, Navarrete (2012), indica que desde la investigación cualitativa, esta técnica atribuye profundidad, ya que busca comprender el significado que tiene un evento específico para los actores involucrados y el entorno. Considerando lo anterior, consiste en un proceso de carácter comunicativo gracias a la interacción de dos o más individuos, a través de la reconstrucción de los procesos, contenidos y vivencias experimentadas por el entrevistado, está técnica es más flexible así como abierta e íntima.

Puede ser considerada como una reunión para intercambiar información entre el entrevistador y el entrevistado. En la entrevista, a través de las preguntas y respuestas, se logra una comunicación además de la construcción conjunta de significados respecto a un tema, en este particular, la gestión del conocimiento en el desarrollo de la capacidad del estudiante de educación primaria de Colombia.

\section{REFLEXIONES FINALES}

Hasta el momento, se han presentado una serie de elementos que permiten apreciar la importancia del flujo del conocimiento, tanto internamente entre los miembros de la organización como la interacción de ese activo interno con el entorno y viceversa, para lograr una organización flexible a los cambios del ambiente.

Puede estimarse, además el valor de la gestión del conocimiento para maximizar la adquisición y desarrollo del conocimiento que necesita la organización educativa para su desempeño exitoso. Del mismo modo, se percibe la gestión de recursos humanos y la gestión de capital humano como elementos esenciales para lograr la participación activa del recurso humano, para poder así incentivar la motivación, utilización y desarrollo del mismo dentro de la organización.

Es importante destacar que el desarrollo del capital humano es el resultado de los beneficios que brindan los procesos de capacitación y desarrollo en las 


\section{CIENCIAMATRIA}

Revista Interdisciplinaria de Humanidades, Educación, Ciencia y Tecnología

Año VI. Vol. VI. N¹0. Enero - Julio 2020

Hecho el depósito de ley: pp201602FA4721

ISSN-L: 2542-3029; ISSN: 2610-802X

Universidad Nacional Experimental Francisco de Miranda (UNEFM). Santa Ana de Coro. Venezuela

\section{Pedro Cruz}

organizaciones. Por ello, es necesario instrumentar a nivel organizacional políticas adecuadas de capacitación y desarrollo de recursos humanos con el objetivo de mantener, incrementar, perfeccionar y aprovechar a niveles más altos los conocimientos esenciales que requiere la organización para su funcionamiento y desempeño.

También es importante destacar que las organizaciones han mostrado un interés creciente en la adopción de modelos para la gestión del conocimiento como vía para incrementar su capacidad innovadora y la creación de ventajas competitivas. Sin embargo, a pesar de la popularidad que ha adquirido recientemente el término aún persiste vacíos conceptuales que dificultan llevar a la práctica de manera exitosa los conceptos y modelos desarrollados hasta el momento.

La falta de aproximaciones prácticas al tema hace difícil justificar soluciones específicas basadas en tecnologías de la información, puesto que adolecen de los elementos blandos necesarios para garantizar una adecuada gestión y absorción de conocimientos en las organizaciones educativas. Es así entonces como la gestión del conocimiento es un proceso ligado estrechamente a las dinámicas de aprendizaje individual y organizacional.

De igual modo puede señalarse que la gestión de conocimiento codificado requiere de procesos de absorción y asimilación con un alto componente motivacionalcognitiva. Este planteamiento hace necesario la elaboración de modelos en los que las soluciones instrumentales y tecnológicas deban estar muy alineadas con una comprensión sistémica de los procesos de aprendizaje y su inserción en las rutinas de la organización.

Por eso se plantea la necesidad de avanzar hacia un mejor entendimiento de los modelos de comprensión, tratamiento y asimilación de la información y conocimientos que conduzcan a formular respuestas más acordes con las necesidades de las organizaciones. 
Revista Interdisciplinaria de Humanidades, Educación, Ciencia y Tecnología

Año VI. Vol. VI. N¹0. Enero - Julio 2020

Hecho el depósito de ley: pp201602FA4721

ISSN-L: 2542-3029; ISSN: 2610-802X

Universidad Nacional Experimental Francisco de Miranda (UNEFM). Santa Ana de Coro. Venezuela

Pedro Cruz

\section{REFERENCIAS CONSULTADAS}

1. Andreu, R y Sieber, S. (2009). La gestión integral del conocimiento y del aprendizaje. Economía Industrial, 326: 63-72.

2. Bell, D (2001) advenimiento de la sociedad post-industrial. Un intento de pronosis social.Madrid. Alianza Editorial. (Original norteamericano 1973).

3. Bisquerra, R. (ed.). (2009). Metodología de la investigación educativa. Madrid: La Muralla.

4. Brooking, A (2009) El capital intelectual: el principal activo de las empresas del tercer milenio. Barcelona: Paidos.

5. Bueno, E. (2010) Dirección estratégica basada en conocimiento: Teoría y práctica de la nueva perspectiva, en Morcillo, P. y Fernández-Aguado, J. (coros): Nuevas claves para la dirección estratégica, Editorial Ariel, Barcelona.

6. Chan, K. y Mauborgne (2007). La estrategia del océano azul: Cómo desarrollar un nuevo mercado donde la competencia no tiene ninguna importancia. Edit. Norma.

7. Denzin, N. K., y Lincoln, Y. S. (2005). The Sage Handbook of Qualitative. Research. London, Inglaterra: Sage.

8. Ianni Gómez., L. (2017). Miramda: Una propuesta educativa emergente desde la investigación. Revista Arbitrada Interdisciplinaria Koinonía, 2(3), 9-30. Recuperado de http://fundacionkoinonia.com.ve/ojs/index.php/revistakoinonia/article/view/49/3 6

9. Klaus, N y Rivas, R (2008) Gestión del conocimiento. Una guía práctica hacia la empresa. Ediciones Díaz de Santos. España.

10. Mayo, A.; Lank, E. (2010). Las organizaciones que aprenden. Ediciones Gesión. Barcelona. España.

11. Morgado, C., Aldana Zavala, J., \& Isea Argüelles, J. (2019). Gerencia transformacional desde el accionar docente directivo para el abordaje del $\begin{array}{llll}\text { Contexto } & \text { Escolar. } & \text { CIENCIAMATRIA, } & 5(9),\end{array}$ https://doi.org/10.35381/cm.v5i9.99

12. Navarrete, J (2012), Investigación cualitativa. Nuevos conceptos y campos de desarrollo. Facultad de Ciencias Sociales, Universidad Nacional Mayor de 
Revista Interdisciplinaria de Humanidades, Educación, Ciencia y Tecnología

Año VI. Vol. VI. N¹0. Enero - Julio 2020

Hecho el depósito de ley: pp201602FA4721

ISSN-L: 2542-3029; ISSN: 2610-802X

Universidad Nacional Experimental Francisco de Miranda (UNEFM). Santa Ana de Coro. Venezuela

Pedro Cruz

San Marcos. Lima - Perú.

13. Nonaka, I. y Takeuchi, H. (2005). The knowledge-creating company. Oxford University Press, Nueva York, USA.

14. Nordan, K. (2009) When your Organization can't get a KM Project. Information Outlook; 9, 3; Academic Research Library.

15.Pavez, A. (2009). La Gestión del Conocimiento en las Organizaciones. Disponible en: http://www.gestiondelconocimiento.com.

16. Prieto, M.I. (2013). Una valoración de la gestión del conocimiento para el desarrollo de la capacidad de aprendizaje en las organizaciones. Propuesta de un modelo integrador. Universidad de Valladolid.

17. Probst, G., Raub, S. y Romhardt, K. (2011). Administre el conocimiento. México DF: Pearson Educación.

18. Strauss, A. y Corbin, J. (2002). Bases de la investigación cualitativa: técnicas y procedimientos para desarrollar la teoría fundamentada. Medellín: Universidad de Antioquia.

19. Tójar, J. (2006) Investigación cualitativa. Comprender y actuar. Madrid: Editorial La Muralla, S.A.

20. Valhondo, D. (2009). Gestión del Conocimiento: del mito a la realidad. Ediciones Díaz de Santos. España.

21. Yuren, M. (2009). Leyes, Teorías y Modelos. México: Trillas.

\section{REFERENCES CONSULTED}

1. Andreu, R and Sieber, S. (2009). The integral management of knowledge and learning. Industrial Economics, 326: 63-72.

2. Bell, D (2001) advent of the post-industrial society. An attempt at social pronosis. Madrid. Editorial Alliance. (Original American 1973).

3. Bisquerra, R. (ed.). (2009). Methodology of educational research. Madrid: The Wall.

4. Brooking, A (2009) Intellectual capital: the main asset of third millennium companies. Barcelona: Paidos. 


\section{CIENCIAMATRIA}

Revista Interdisciplinaria de Humanidades, Educación, Ciencia y Tecnología

Año VI. Vol. VI. N¹0. Enero - Julio 2020

Hecho el depósito de ley: pp201602FA4721

ISSN-L: 2542-3029; ISSN: 2610-802X

Universidad Nacional Experimental Francisco de Miranda (UNEFM). Santa Ana de Coro. Venezuela

\section{Pedro Cruz}

5. Bueno, E. (2010) Strategic management based on knowledge: Theory and practice of the new perspective, in Morcillo, P. and Fernández-Aguado, J. (choirs): New keys for strategic management, Editorial Ariel, Barcelona.

6. Chan, K. and Mauborgne (2007). The blue ocean strategy: How to develop a new market where competition is of no importance. Edit Rule.

7. Denzin, N. K., and Lincoln, Y. S. (2005). The Sage Handbook of Qualitative. Research London, England: Sage.

8. Ianni Gómez., L. (2017). Miramda: An educational proposal emerging from research. Interdisciplinary Arbitrated Review Koinonía, 2 (3), 9-30. Recovered from http://fundacionkoinonia.com.ve/ojs/index.php/revistakoinonia/article/view/49/ 36

9. Klaus, $\mathrm{N}$ y Rivas, $\mathrm{R}$ (2008) Knowledge management. A practical guide to the company. Díaz de Santos editions. Spain.

10. May, A.; Lank, E. (2010). The organizations that learn. Editions Session. Barcelona. Spain.

11. Morgado, C., Aldana Zavala, J., \& Isea Argüelles, J. (2019). Transformational management from the directive teaching actions to address the School Context. SCIENCE, 5 (9), 48-72. https://doi.org/10.35381/cm.v5i9.99

12. Navarrete, J (2012), Qualitative research. New concepts and fields of development. Faculty of Social Sciences, National University of San Marcos. Lima Peru.

13. Nonaka, I. and Takeuchi, H. (2005). The knowledge-creating company. Oxford University Press, New York, USA.

14.Nordan, K. (2009) When your Organization can't get a KM Project. Information Outlook; 9, 3; Academic Research Library.

15. Pavez, A. (2009). Knowledge Management in Organizations. Available at: http://www.gestiondelconocimiento.com.

16. Prieto, M.I. (2013). An assessment of knowledge management for the development of learning capacity in organizations. Proposal of an integrative model. University of Valladolid.

17. Probst, G., Raub, S. and Romhardt, K. (2011). Manage knowledge. Mexico City: Pearson Education. 


\section{CIENCIAMATRIA}

Revista Interdisciplinaria de Humanidades, Educación, Ciencia y Tecnología

Año VI. Vol. VI. N¹0. Enero - Julio 2020

Hecho el depósito de ley: pp201602FA4721

ISSN-L: 2542-3029; ISSN: 2610-802X

Universidad Nacional Experimental Francisco de Miranda (UNEFM). Santa Ana de Coro. Venezuela

\section{Pedro Cruz}

18. Strauss, A. and Corbin, J. (2002). Basis of qualitative research: techniques and procedures to develop grounded theory. Medellín: University of Antioquia.

19.Tójar, J. (2006) Qualitative research. Understand and act. Madrid: Editorial La Muralla, S.A.

20. Valhondo, D. (2009). Knowledge Management: from myth to reality. Díaz de Santos editions. Spain.

21. Yuren, M. (2009). Laws, Theories and Models. Mexico: Threshing.

(C2020 por los autores. Este artículo es de acceso abierto y distribuido según los términos y condiciones de la licencia Creative Commons Atribución-NoComercial-Compartirlgual 4.0 Internacional (CC BY-NC-SA 4.0) (https://creativecommons.org/licenses/by-nc-sa/4.0/). 